\title{
Data Analytics for Performance Monitoring of Gas Turbine Engine
}

\author{
Yuan Liu ${ }^{1}$, Avisekh Banerjee ${ }^{2}$, Thambirajah Ravichandran ${ }^{3}$, Amar Kumar $^{4}$, and Glenn Heppler ${ }^{5}$ \\ ${ }^{1,4}$ Tecsis Corporation, 201-203 Colonnade Road, Ottawa, ON, K2E 7K3, Canada \\ yliu@tecsis.ca \\ amar@tecsis.ca \\ ${ }^{2}$ Life Prediction Technology Inc., 1010 Polytek Street, Ottawa, ON, K1J 9J1, Canada \\ banerjeea@lifepredictiontech.com \\ ${ }^{3,5}$ Department of Systems Design Engineering, University of Waterloo, Waterloo, ON, N2L 3G1, Canada \\ travicha@uwaterloo.ca \\ heppler@uwaterloo.ca
}

\begin{abstract}
Performance analysis of a low power rating and partially loaded industrial gas turbine engine (GTE) was carried out by using a model-free data analytic approach. By adopting an efficient input selection method, several performance indices (PI) are proposed to quantify the performance of the GTE. These indices are extracted using engine operating data related to power output and parameters related to fuel consumption, and validated with engine performance monitoring measurements for a three year period corresponding to one time between overhaul intervals. The dependency of the PIs on ambient temperature has been studied by using linear and polynomial fitting curves. Then novel methods are introduced for analysis of short-term and long-term performance deterioration arising from compressor fouling and structural degradation respectively. The results have clearly shown the ability of the proposed PIs to detect short-term compressor fouling as well as long-term performance deterioration, which is directly relevant to the Prognostics and Health Management of gas turbine engine.
\end{abstract}

\section{INTRODUCTION}

Gas turbine engines (GTE) play key roles in the fields of power generation, transportation, and aerospace. Gas turbines are popular due to their attractive properties such as high power to weight ratio, compactness, ease of installation and environmental friendliness (low pollution products).

The performance of GTE gradually deteriorates during the operation even under normal engine operating conditions.

Yuan Liu et. al. This is an open-access article distributed under the terms of the Creative Commons Attribution 3.0 United States License, which permits unrestricted use, distribution, and reproduction in any medium, provided the original author and source are credited.
There are two major mechanisms contributing to the performance deterioration (Diahunchak, 1991; Hanachi, 2015). One is the rapid (short-term) deterioration due to fouling and congestions of the particles in the air and in the combustion gases on the rotating and stationary blades. The 'fouling' of the surfaces of upstream sections results in varying degrees of performance deterioration of the GTE. This type of performance deterioration is recoverable with cleaning/washing (Meher-Homji, 2009). Another type of deterioration is slower and non-recoverable with washing. It occurs due to structural degradation of the GTE parts such as flow path damage, surface erosion and corrosion, distortion, etc. The resulting performance deterioration is irrecoverable by cleaning and washing, but will get worse with usage, unless the degraded parts are repaired or replaced (Kurz and Brun, 2009).

In the application of gas turbines, the main concerns of gas turbine users are availability, reliability and maintenance costs. In order to have high levels of availability and reliability of gas turbines, effective maintenance is essential, and the strategies for maintenance are very important. In conventional and preventative GTE maintenance strategies, pre-scheduled overhauls and maintenance services are carried out regardless of the health condition of the gas turbine engines. A GTE may be sent for unnecessary maintenance when it is still in good health condition or it may fail before a scheduled overhaul. As a consequence, the availability of the GTEs decrease and the costs of the maintenance may increase. Condition-based maintenance $(\mathrm{CBM})$ is an effective way to improve engine availability and reduce maintenance costs by adopting gas turbine health information obtained from diagnostic and prognostic analyses (Li and Nilkitsaranont, 2009). 
The prediction of GTE performance deterioration and future structural health state is highly complicated and challenging due to the structural complexity, non-stationary operating conditions and great uncertainty associated with gas turbine design, manufacturing, ambient and environment condition, etc. Different prognostic techniques have been investigated by many researchers and the types of the techniques can be summarized into two groups: model-based prognostics (Kacprzynski et al., 2001; Carter, 2005; Li and Lee, 2005; Sun et al., 2012; Coble and Hines, 2013; Feng et al., 2013) and data-driven approaches (Hanachi, et al., 2012; Rai and Kohli, 2012).

GTE fault and diagnostics is an essential step to perform effective prognostic analysis. Many automatic diagnostic techniques have been developed in the past, which can be categorized into three main types (Li, 2002): Model-based methods (Feldman et al., 2013), Data-driven methods (Namburu et al., 2006) and Hybrid approach, which are a combination of both model-based and data-driven models (Yu et al., 2007; Luo et al., 2010).

Each of the reported diagnostic techniques, however, has its own benefits and limitations. Also, almost no analysis and research has been reported by other researchers before for gas turbine performance. There is still a need to study the effect of ambient temperature and fuel consumption on short-term fouling as well as long-term performance deterioration. Existing Model-free methods for diagnostics and prognostics of gas turbine degradation state are based on artificial Intelligence (AI) and statistical methods. These methods usually require enormous amount of computing time, highly significant data and often very expensive. Simple yet insightful data-analytics based model-free approaches are also desirable for easy implementation in an industrial setting and real-time application.

A simple, direct and thermodynamic model-free analysis methodology for Gas turbine engine (GTE) performance evaluation had been reported in our preliminary work (Yuan et al., 2017). A performance index (ratio of power generation to fuel consumption) was proposed as the metric for monitoring the engine performance. The motivation behind this work is to extend the study to propose more performance indices by taking into account more parameter combinations and apply them to the investigation of short-term and longterm performance deterioration patterns. These analyses and results are crucial for assessing both the prediction of engine performance with the output power, efficiency, exit gas temperature (EGT) and its reliability.

The aims of this research work include:

- Study the feasibility of introducing additional performance measurement indices derived based on model-free data analysis.

- Investigate the effect of ambient temperature on the performance indices.
- Identify the rapid and short-term performance deterioration patterns, i.e., compressor fouling-washing effects using the proposed performance indices.

- Identify the subtle and long-term performance deterioration patterns (in terms of decrease of the efficiency) of the GTE during the operating time, using the proposed performance indices.

\section{DATA anAlytics APPROACH}

All the input engine parameters have individual and combined effects on the GTE performance and output engine parameters. Attempts are made here to isolate the effects of individual input parameters or phenomenon for a meaningful analysis and functional relationships of the selected significant input parameters to the performance related output parameters. Engine operating and output data measured from a $4 \mathrm{MW}$ power generation turbine was collected over a three year period corresponding to one time between overhaul. The data collected from the beginning of this period corresponds to a freshly built engine, while the data collected near the end of this period corresponds to a degraded engine that needs overhauling. A data analytics approach is proposed that consists of the following steps.

Step 1: Collect and clean data

In this step, the measured data is collected from the gas turbine and the data is cleaned by filtering any measurement noise. The data is arranged in an hourly, daily and monthly basis to make observations over different time periods.

Step 2: Define the performance indices

In this step, several performance indices are proposed and are supported by input selection analysis methods. Proposed performance indices are based on physics and indicate the amount of fuel consumed to generate unit power and are proportional to the efficiency of the gas turbine.

Step 3: Study the effect of ambient temperature

The effect of the ambient temperature is studied on the performance indices to understand and eliminate the effect of the ambient conditions on the performance.

Step 4: Study the short-term performance deterioration The identification of the loss of power due to shortterm performance deterioration caused by compressor fouling is studied with the proposed performance indices by taking data samples before and after washing the compressor.

Step 5: Study the long-term performance deterioration In this step, the identification of the long-term performance deterioration is studied with the proposed performance indices. This slow-moving and irrecoverable performance deterioration occurs 
due to structural degradation of the parts such as erosion and corrosion processes.

\section{DATA COLLECTION AND TRENDS}

\subsection{Ambient Data}

Ambient data were collected from Environment Canada and the three ambient parameters of interest include ambient temperature $\left(\mathrm{T}_{\mathrm{amb}}\right)$, relative humidity $\left(\mathrm{H}_{\mathrm{rel}}\right)$ and ambient pressure $\left(\mathrm{P}_{\mathrm{amb}}\right)$, as shown in Figure 1. The compressor of the power plant is located outside, thus $\mathrm{T}_{\mathrm{amb}}$ is assumed to be the temperature of air into the compressor. It can be seen that $\mathrm{T}_{\mathrm{amb}}$ shows clear seasonal variation within the range of $240 \mathrm{~K}$ to $310 \mathrm{~K}$ while variations in $\mathrm{P}_{\mathrm{amb}}$ over three years are found to be less than $5 \%$ and so are considered to be insignificant for any meaningful dependency analysis. The $\mathrm{H}_{\text {rel }}$ values may be seen to be distributed incoherently with the seasons or month of the year, unlike the distributions for $\mathrm{T}_{\mathrm{amb}}$. $\mathrm{P}_{\mathrm{amb}}$ and $\mathrm{H}_{\mathrm{rel}}$ are not considered in our present analysis because of the lack in any definite trend and variations.
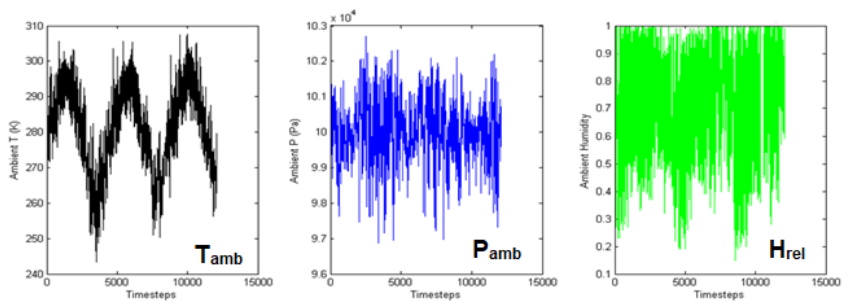

Figure 1. Important ambient parameters for three years

\subsection{Gas Turbine Engine Data}

The data analyzed in this work is based on the field data as obtained from a low power gas turbine engine from a power generation turbine located in Ottawa, Canada. The data was collected and monitored at a frequency of every 2 hours. Performance analysis is carried out using three years of operation-maintenance cycle data. Analysis of the continuous data stream (time series) provides significant clues in advance about any developing faults or deviation in the operating parameters like pressure, temperature, power output that may be correlated to any physical damage (e.g. cracks, wear) in the hot gas path components.

The generated power is usually a function of the mass flow of air, the temperature difference and the specific heat of air. On the other hand, the thermal health condition in a GTE is measured by the EGT, which is a measure of the engine's efficiency in producing its design level of power. The EGT gives an indication of performance deterioration and its prediction in advance estimates the remaining Timebetween-overhauls (TBO).

Power generation in GTE depends on the quantity, quality and type of fuel used and so the performance and efficiency strongly depends on fuel consumption. The supply and dayto-day demand of power can be met with the control of fuel (Gas) consumption. The fuel consumption (Gas) is adjusted at the end of the day for the GT (Gas Turbine) system and is maintained constant for a day ( $24 \mathrm{hrs})$, which is recorded as Gas and used in present work. Also, the other two important parameters related to the fuel consumption.

\subsection{Trends of Data}

An average of 12 GTE measurements and ambient parameters are calculated to obtain daily values of the parameters in the present analysis. Figure 2 displays the trend and distribution of $\mathrm{T}_{\mathrm{amb}}$ data and several important GTE data in normalized scale, including Power, EGT, Fuel Angle (FA), Fuel Pressure (FP) and Fuel consumption (Gas). Means of the respective data for each day are determined to display as shown for a three year period corresponding to a complete TBO. The $\mathrm{T}_{\mathrm{amb}}$ data is most symmetrically and uniformly distributed with peak during the summer months and lowest during the winter months; while the EGT shows the opposite trend with some degree of irregularities and disturbance. Power appears to shows similar fluctuations in terms of peaks and valleys over a year, though these do not match precisely with those observed for $\mathrm{T}_{\mathrm{amb}}$ on the time scale ( $\mathrm{x}$-axis). Also, Gas seems to closely follow the trend of Power while the variation of the Power is less than that of Gas.
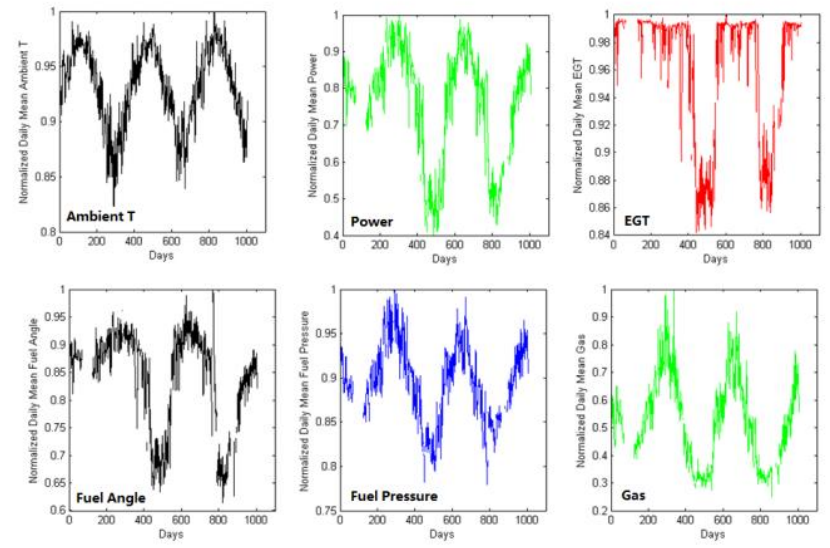

Figure 2. Daily trend of $\mathrm{T}_{\mathrm{amb}}$ and important GTE parameters for three years

\section{PERFormanCE INDICES}

\subsection{Proposed Performance Indices}

In this work, performance indices (PI) are parameters that are candidates to quantify the performance of the GTE. These can be directly the engine measurements, their normalized values or combinations of different measurements. The intent is to preserve some physical insight such that causes for change in the performance observed through the PIs can be better understood and explained. Besides the PI01, which has been proposed in our previous research (Yuan et. al, 2017), 
five new PIs are proposed in this analysis by using combination of input data. The proposed PIs are defined in Table 1. PI10 and PI11 are using a combination of fuel angle and fuel pressure to represent the gas consumption. For PI12 to PI14, the density of fuel gas is taken into account. Assuming the fuel gas behaves as an ideal gas, then there is the ideal gas law as shown in equation 1:

$$
P v=n R T
$$

where $P$ is the pressure, $v$ is the volume, $n$ is the amount of substance of the gas (in moles), $R$ is the universal gas constant (8.314 $\mathrm{J} \mathrm{K}-1 \mathrm{~mol}-1$ ) and $T$ is the absolute temperature (in $K$ ). Thus, the density of the fuel gas is:

$$
\rho=\frac{P}{n R T}
$$

Since both $n$ and $R$ are constants for an ideal gas, the density of the fuel gas can be expressed as:

$$
\rho \propto \frac{P}{T}
$$

Thus, the three new performance indices including the effect of density of fuel gas are proposed as shown in the second group of Table 1.

Table 1 List of proposed Performance Indices.

\begin{tabular}{|l|l|}
\hline PI & Definition \\
\hline PI01 & Power $/$ Gas \\
\hline PI02 & Power $/ F A$ \\
\hline PI05 & EGT $/ F A$ \\
\hline PI10 & Power $/(F A \times F P)$ \\
\hline PI11 & EGT $/(F A \times F P)$ \\
\hline PI12 & Power $/(G A S \times F P / T)$ \\
\hline PI13 & Power $/\left(F A \times F P^{2} / T\right)$ \\
\hline PI14 & EGT $/\left(F A \times F P^{2} / T\right)$ \\
\hline
\end{tabular}

\subsection{Input Selection Analysis}

All the input parameters have individual and combined effects on the performance and output parameters of the GTE. Attempts are made here to isolate effects of individual input parameters for a meaningful analysis and functional relationships of the desired input and output parameters.

In order to support the choices for the new performance indices as proposed in Table 1, input selection analysis is performed here using correlation analysis to determine the influence of each set of input parameters on two output parameters, namely the Power and EGT. The objective of this input selection analysis is to determine the most influential individual input parameters on the desired performance and output parameters such as Power and EGT.

It is well known that correlation analysis plays a major role in input ranking based input selection analysis (Guyon and
Elisseeff, 2003). The correlation criteria based input ranking method uses the sample correlation coefficient ( $r$ - also known as the Pearson correlation coefficient when applied to a sample) as the measure of relevancy between the individual input and the output (Guyon and Elisseeff, 2003). Using this correlation coefficient as an input ranking criterion will in effect implement a ranking according to the goodness of linear fit of the output parameter with individual input parameters. In order to compute the sample correlation coefficient (r), the Matlab function corrcoef can be used to calculate a matrix of correlation coefficients for a given input data matrix $\mathrm{X}$ and a target (output) vector $\mathrm{y}$ as follows: $\mathrm{R}=$ $\operatorname{corrcoef}([\mathrm{y} \mathrm{X}])$, where the input data is represented by the matrix $X$, in which each row is an observation and each column is an input parameter, and the output data is represented by the vector $\mathbf{y}$. Any off-diagonal element of $\mathbf{R}$ provides the sample correlation coefficient between the corresponding two column vectors.

A data set with 120 data samples from the GTE washout operation is considered here for the correlation analysis. The following set of input parameters, namely, Gas, FA, FP, Gas $\times F A, G a s \times F P, F A \times F P, G a s \times F P / T$, and $F A \times F P^{\wedge} 2 / T$ along with output parameters Power and EGT are considered for this correlation analysis. The correlation coefficients between the above inputs and the Power and EGT outputs are given in Table 2. Scatter plots of these outputs versus the abovementioned inputs are shown in Figure 3 to Figure 5.

Table 2 Correlations between input and output parameters.

\begin{tabular}{|l|l|l|}
\hline & Power & EGT \\
\hline$G A S$ & 0.9160 & 0.7713 \\
\hline$F A$ & 0.9574 & 0.9261 \\
\hline$F P$ & 0.9155 & 0.7568 \\
\hline$G A S \times F A$ & 0.9453 & 0.8129 \\
\hline$G A S \times F P$ & 0.9163 & 0.7592 \\
\hline$F A \times F P$ & 0.9913 & 0.9126 \\
\hline$G A S \times F P / T$ & 0.9090 & 0.7428 \\
\hline$F A \times F P^{2} / T$ & 0.9831 & 0.8561 \\
\hline
\end{tabular}

High correlations values between the Power and EGT outputs and the selected inputs as shown in Table 2 support the choice of the proposed new performance indices as given in Table 1. Scatter plots shown in Figures $3-5$ also illustrate these high correlations between the outputs of interest and the selected inputs, notably Power and $F A, F A \times F P$, and $F A \times F P^{2} / T$. One can also observe the flattening of EGT curves against the selected inputs in the above figures that indicate the influence of the control system that regulates the EGT at certain constant values. This artifact in the trend of EGT curves due to the controller effect decreases the effectiveness of PIs involving EGT for performance monitoring. 

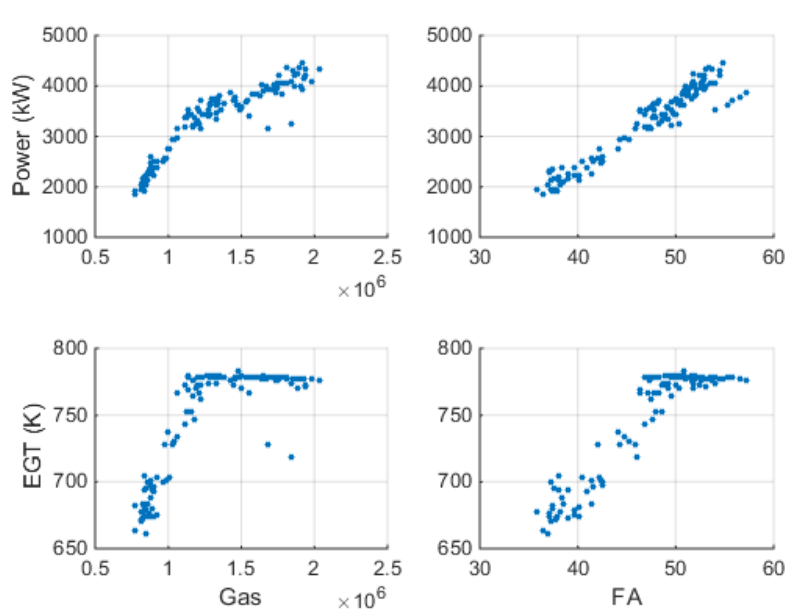

Figure 3. Scatter plots between power and EGT outputs and Gas $\left(f t^{3}\right)$ \& FA (degree) inputs.
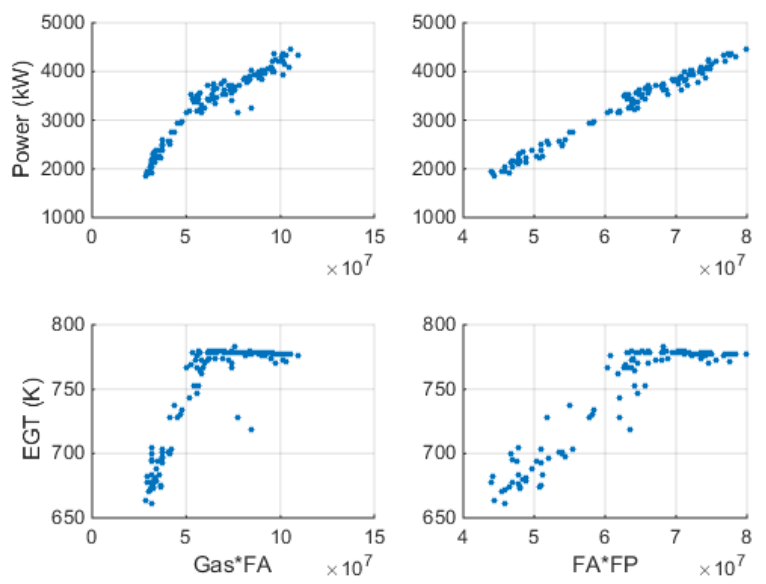

Figure 4. Scatter plots between Power \& EGT outputs and $\mathrm{Gas} \times \mathrm{FA}\left(\mathrm{ft}^{3} \times\right.$ degree $) \& \mathrm{FA} \times \mathrm{FP}($ degree $\times \mathrm{Pa})$ inputs.
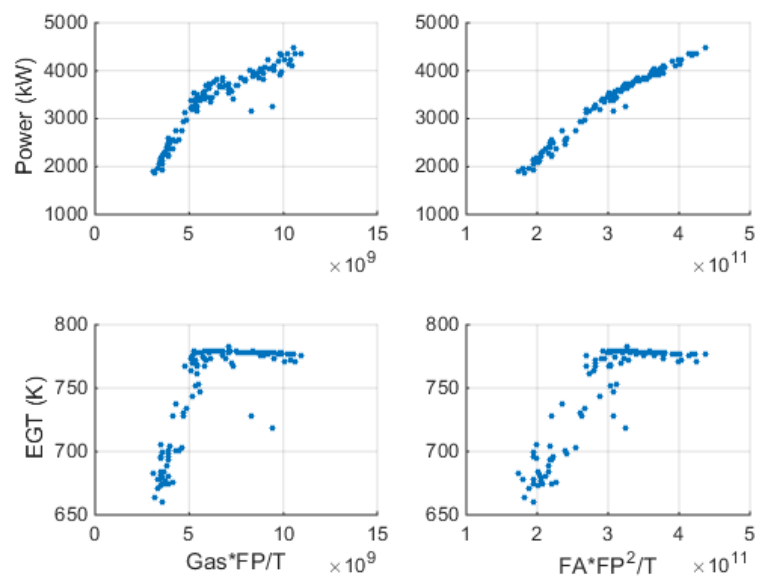

Figure 5. Scatter plots between power \& EGT outputs and Gas $\times \mathrm{FP} / \mathrm{T}\left(f t^{3} \times P a / K\right) \& \mathrm{FA} \times \mathrm{FP}^{2} / \mathrm{T}\left(\right.$ degree $\left.\times P a^{2} / K\right)$ inputs.

\section{EFFECT OF AMBIENT TEMPERATURE ON PI}

As reported in our previous research, $\mathrm{T}_{\mathrm{amb}}$ has a noticeable influence on GTE operating parameters. This arises from the seasonal variations in the ambient temperature from 240 to $310 \mathrm{~K}$. So it becomes important to study and eliminate the effect of the ambient temperature on the PIs before using them to quantify performance deterioration. Figure 6 presents the dependency of the PIs on $\mathrm{T}_{\mathrm{amb}}$. It can be seen that the three PIs are related to Power except for PI02 and PI10 increase with increasing $\mathrm{T}_{\mathrm{amb}}$ until $288 \mathrm{~K}$. The PIs related to Power reach a peak when $\mathrm{T}_{\mathrm{amb}}$ is around $288 \mathrm{~K}$, which matches the fact that for this turbine, the value of $\mathrm{T}_{\mathrm{amb}}$ at the design point is $288.15 \mathrm{~K}$. As discussed in the previous section, both $\mathrm{PW}_{\text {out }}$ and Gas decrease with increasing $\mathrm{T}_{\mathrm{amb}}$. And the variation of $\mathrm{PW}_{\text {out }}$ is less than that of the Gas when $\mathrm{T}_{\mathrm{amb}}$ is below $288 \mathrm{~K}$. Thus the gas turbine power plant reaches its highest efficiency and then begins to decrease. One of the possible reasons for it is the specific fuel consumption increases with the increase of $\mathrm{T}_{\mathrm{amb}}$ due to the fuel gases losses. Also, when less than full power is required from a gas turbine, the output is reduced by lowering the turbine inlet temperature. In addition to reducing power, this change in operating conditions also reduces efficiency.

After the gas has passed through the turbine, it is discharged through the exhaust. Though most of the chemical energy is converted to mechanical energy by the turbine, a significant amount of power remains in the exhaust gas. Higher EGT indicate that more energy from the fuel is being wasted rather than being converted to the power (or mechanical energy sent to the propeller). So the PIs related to the EGT, should be considered to inversely relate to efficiency of the GTE, which can be seen from the inverse trends of PI11 and PI14 illustrated in Figure 6.

Polynomial models are among the most frequently used empirical models for curve fitting, and it is adopted in the present research. A polynomial function is one that has the form:

$$
y=\alpha_{n} x^{n}+\alpha_{n-1} x^{n-1}+\cdots+\alpha_{2} x^{2}+\alpha_{1} x+\alpha_{0}
$$

The linear functional relationships between GTE parameters and $\mathrm{T}_{\mathrm{amb}}$ can be represented as:

$$
P I=\alpha_{1} \times T_{a m b}+\alpha_{0}
$$

The $2^{\text {nd }}$ degree polynomial functional (quadratic) relationships between GTE parameters and $T_{a m b}$ can be represented as:

$$
P I=\alpha_{2} \times T_{a m b}^{2}+\alpha_{1} \times T_{a m b}+\alpha_{0}
$$

The coefficients and $R^{2}$ value for first and $2^{\text {nd }}$ degree Polynomial Empirical relationships of PIs with $\mathrm{T}_{\mathrm{amb}}$ are listed in Table 3 and Table 4, respectively. For all PIs, the $\mathrm{R}^{2}$ values of less than 0.1 indicate that when $\mathrm{T}_{\mathrm{amb}}$ is higher than $288 \mathrm{~K}$, the linear regression model is not fitting the data points very 
well for this ambient temperature range. From the figures and tables, it can be seen that the 2nd degree polynomial (quadratic) method provides better fitting results whose $\mathrm{R}^{2}$ values are higher than those of linear fitting curves. The
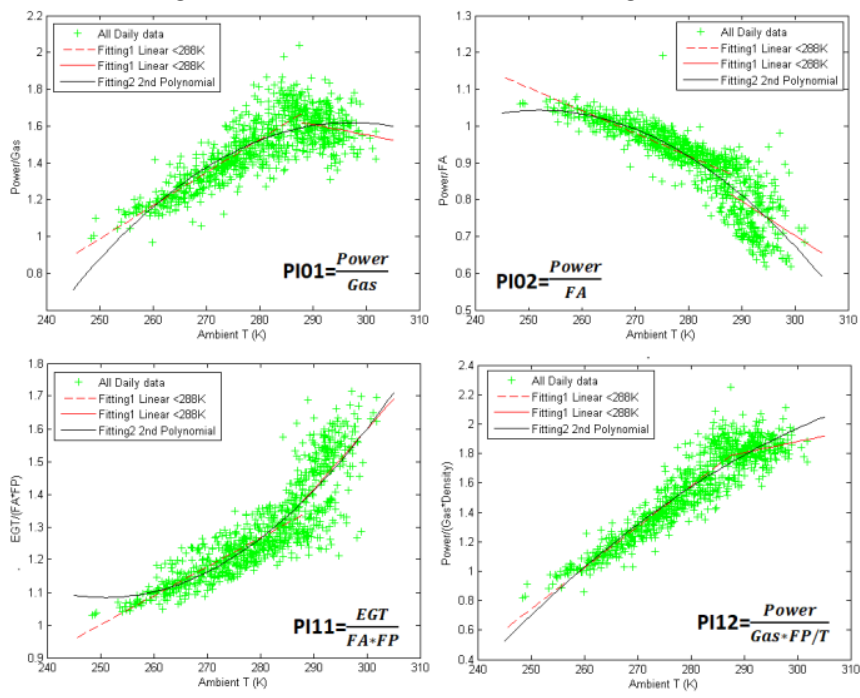

variation of the PIs with the ambient temperature shall be systematically eliminated while using them for estimating the performance in the subsequent sections.
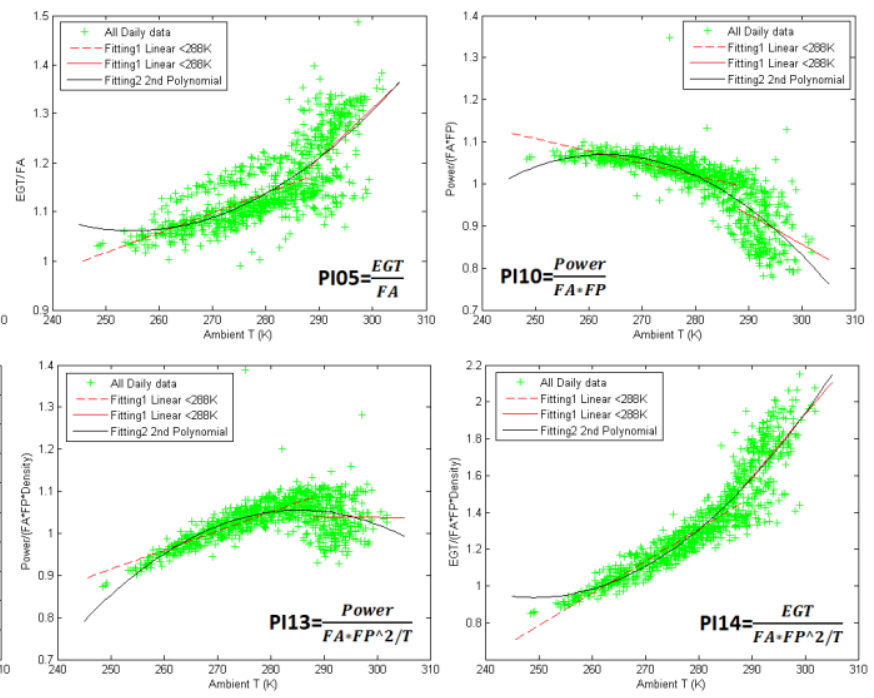

Figure 6. Dependency of new PIs on $\mathrm{T}_{\mathrm{amb}}$

Table 3 Coefficients and $R^{2}$ for linear relationship of PIs with $\mathrm{T}_{\mathrm{amb}}$

\begin{tabular}{|l|l|l|l|l|l|l|}
\hline \multirow{2}{*}{ PI } & \multicolumn{3}{|l}{$\mathbf{T}_{\mathrm{amb}}<\mathbf{2 8 8 K}$} & \multicolumn{2}{l|}{$\mathbf{T}_{\mathrm{amb}} \mathbf{2 8 8 K}$} \\
\cline { 2 - 8 } & $\boldsymbol{\alpha}_{\mathbf{1}}$ & $\boldsymbol{\alpha}_{\mathbf{0}}$ & $\mathbf{R 2}$ & $\boldsymbol{\alpha}_{\mathbf{1}}$ & $\boldsymbol{\alpha}_{\mathbf{0}}$ & $\mathbf{R}^{\mathbf{2}}$ \\
\hline PI01 & 0.0180 & -3.5138 & 0.7180 & -0.0056 & 3.2235 & 0.0314 \\
\hline PI02 & -0.0062 & 2.6471 & 0.7030 & -0.0093 & 3.4989 & 0.1227 \\
\hline PI05 & 0.0040 & 0.0064 & 0.4363 & 0.0102 & -1.7464 & 0.1621 \\
\hline PI10 & -0.0029 & 1.8379 & 0.4250 & -0.0071 & 2.9952 & 0.1006 \\
\hline PI11 & 0.0089 & -1.2156 & 0.6930 & 0.0183 & -3.9017 & 0.2231 \\
\hline PI12 & 0.0279 & -6.2254 & 0.8386 & 0.0075 & -0.3660 & 0.0597 \\
\hline PI13 & 0.0045 & -0.2183 & 0.6683 & -0.0002 & 1.0855 & $9.47 \mathrm{E}-05$ \\
\hline PI14 & 0.0175 & -3.5919 & 0.8340 & 0.0342 & -8.3151 & 0.3115 \\
\hline
\end{tabular}

Table 4 Coefficients and $R^{2}$ for 2 nd degree polynomial relationship of PIs with $\mathrm{T}_{\mathrm{amb}}$

\begin{tabular}{|c|r|r|r|r|}
\hline PI & \multicolumn{1}{c|}{$\boldsymbol{\alpha}_{\mathbf{2}}$} & \multicolumn{1}{c|}{$\boldsymbol{\alpha}_{\mathbf{1}}$} & \multicolumn{1}{c|}{$\boldsymbol{\alpha}_{\mathbf{0}}$} & \multicolumn{1}{c|}{$\mathbf{R}^{\mathbf{2}}$} \\
\hline PI01 & $-3.35 \mathrm{E}-04$ & 0.1988 & -27.9075 & 0.6649 \\
\hline PI02 & $-1.61 \mathrm{E}-04$ & 0.0813 & -9.2100 & 0.777 \\
\hline PI05 & $1.22 \mathrm{E}-04$ & -0.0621 & 8.9800 & 0.609 \\
\hline PI10 & $-1.75 \mathrm{E}-04$ & 0.0919 & -11.0136 & 0.6762 \\
\hline PI11 & $2.15 \mathrm{E}-04$ & -0.1077 & 14.6037 & 0.7733 \\
\hline PI12 & $-1.82 \mathrm{E}-04$ & 0.1256 & -19.3141 & 0.859 \\
\hline PI13 & $-1.61 \mathrm{E}-04$ & 0.0919 & -12.0608 & 0.4701 \\
\hline PI14 & $3.86 \mathrm{E}-04$ & -0.1924 & 24.9037 & 0.862 \\
\hline
\end{tabular}

\section{SHORT-TERM PERFORMANCE DETERIORATION}

The fouling of axial flow compressors causes a major loss of performance during the engine operation. It however results in a short-term deterioration in the performance that can be usually recovered though compressor washes but leads to additional downtime. Hence optimal scheduling of compressor wash becomes important for gas turbine operators especially in a highly competitive power market. It is also significant in the oil and gas generation market where a loss in gas turbine output directly affects the production.

The fouling (operational) cycle between two subsequent washes shows changes in the GTE measurement. Hence a data set for just 5 days (i.e. 120 hrs.) at the beginning as well as at the end of a cycle were selected and combined to create a separate group for analyzing fouling. It is assumed that the data set collected from the beginning of the cycle corresponds to a fouling free condition of the compressor. The data from the last few days of the cycle corresponds to an adequately fouled condition of the rotor. This approach facilitates the meaningful comparison of fully fouled and clean or no fouling conditions. For each wash time, the after-wash value is compared with before-wash value, and the absolute value and the percentage of difference between them is calculated.

$$
\begin{array}{rr}
\Delta_{i}=P I_{\text {After }, i}-P I_{\text {Before }, i} \quad(\mathrm{j}=2,3,4, \ldots, 12) \\
\Delta_{i} \%=\frac{\Delta_{i}}{P I_{\text {After }, i}} \quad(\mathrm{j}=2,3,4, \ldots, 12)
\end{array}
$$

in which, $i$ is the number of the wash.

Figure 7 shows the short-term difference for each PI. It can be seen that for PIs related to Power (PI01, PI10, PI12, and PI13), almost all the $\Delta_{i}$ values are positive, which indicates that after-wash values are higher than the before-wash values for each wash time. All PIs related to Power show very good wash effect and short-term trends when this approach is adopted, except for PI13. The possible reason may be due to 
the fuel density that we assumed for an idea gas. In future, more work will be performed to investigate the density of the real fuel gas.

Also, for PIs related to EGT (PI05, PI11 and PI14), all the $\Delta_{i}$ values are negative, which indicates that after-wash values are lower than before-wash values for each wash time. As discussed in the previous section, higher EGT indicate that more energy from the fuel is being wasted rather than being converted to power. Hence the short-term trends for the two PIs related to EGT (PI11 and PI14), this method also provide negative difference. It is also notable that curves of the shortterm difference of PI05, PI11 and PI14 show monotonically increasing trends, indicating the decrease in the effect of washing to recover the performance losses. There is a sharp drop of the short-term difference at the tenth wash, which indicates a dramatic rise of performance after the tenth wash. One possible reason for it is that some servicing of the turbine was performed along with the compressor wash.
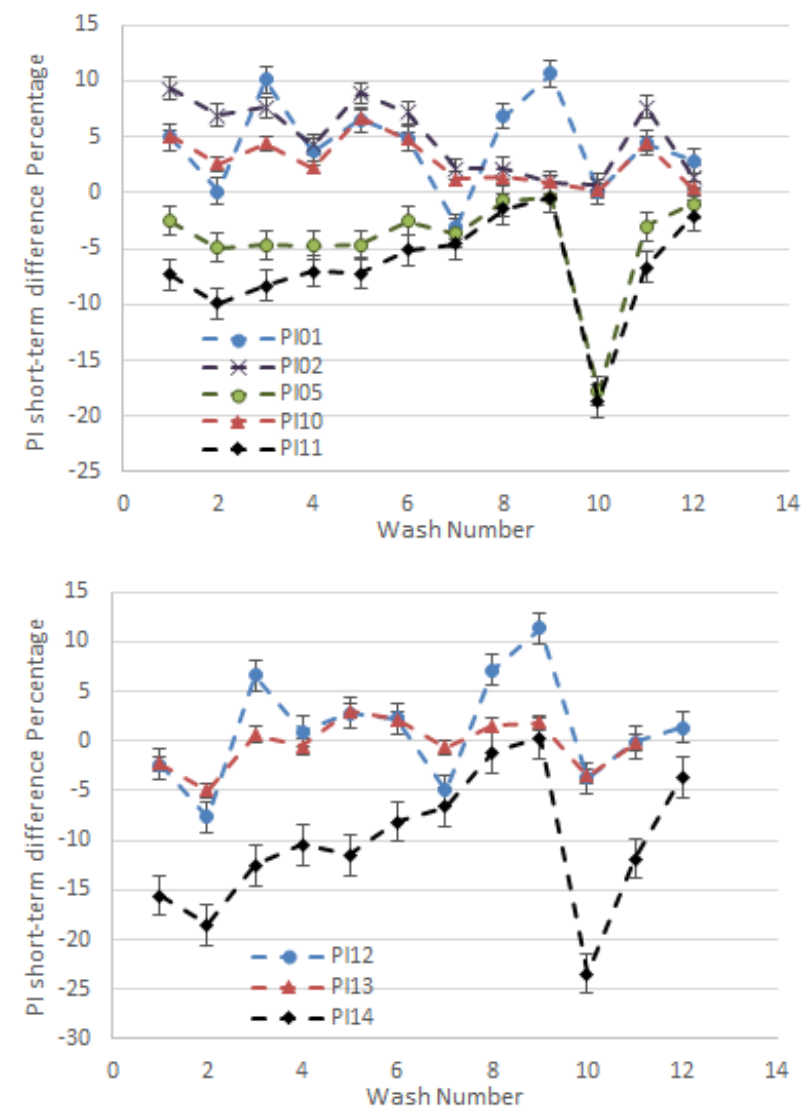

Figure 7. Short-term patterns for PIs

\section{LONG-TERM PERFORMANCE DETERIORATION}

The long-term performance deterioration is estimated by eliminating the short-term performance deteriorations from the GTE measurements. This deterioration corresponds to the non-recoverable usage-dependent structural degradations cumulated with usage especially in the hot gas path components. In this work, a direct approach is developed and adopted for estimating long-term performance deterioration patterns, in which, the difference between PI after each wash with the PI after the first wash is calculated and accumulated, as shown in following equations:

$$
\begin{gathered}
\Delta_{j}=P I_{A f t e r, j}-P I_{A f t e r, 1} \quad(j=2,3,4, \ldots, 12) \\
\text { Accumulated } \Delta_{j} \%=\sum_{j=2}^{n} \frac{\Delta_{j}}{P I_{A f t e r, j}} \quad(\mathrm{j}=2,3,4, \ldots, 12)
\end{gathered}
$$

in which $j$ is the number of the wash.

This method directly compares the after wash values of the PI for each wash time, eliminating the effect of the short-term deterioration and the influence of the ambient temperature.
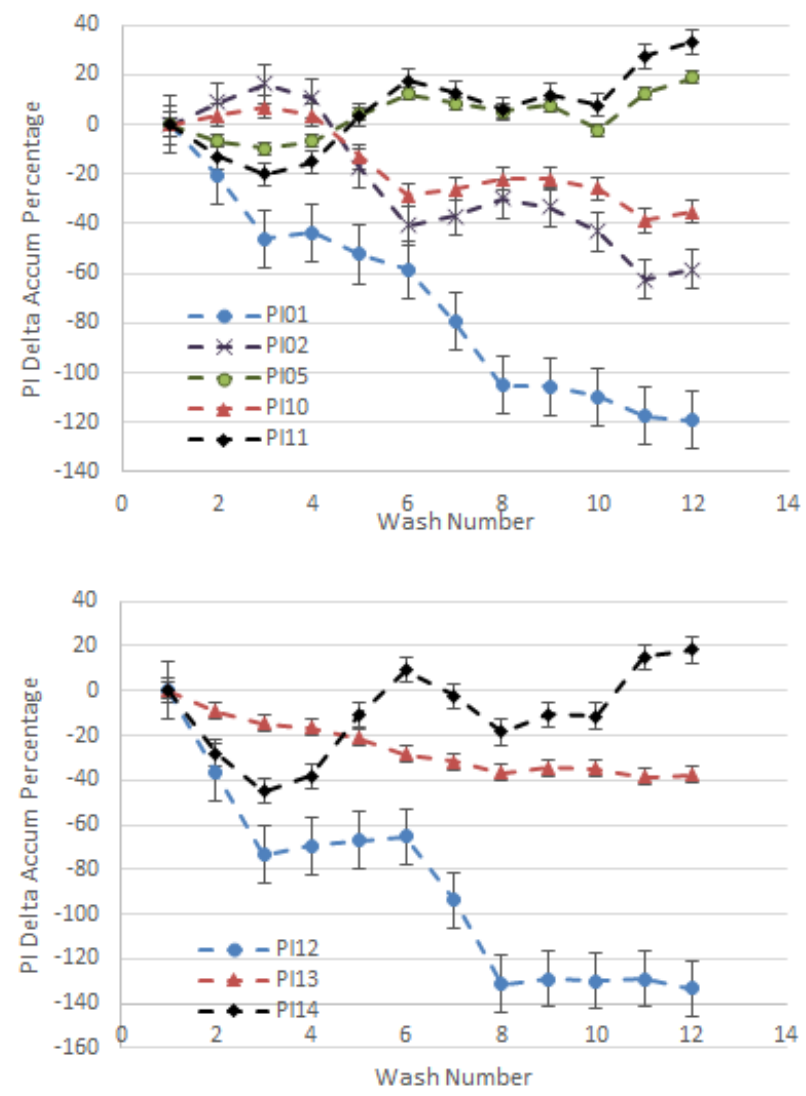

Figure 8. Long-term trends for new PIs

The accumulated differences between the PIs after every wash and after the first wash are plotted in Figure 8. It can be seen that for PIs related to Power, the accumulations are mostly negative, irrespective of the density of fuel gas. This suggests that the efficiency of the GTE decreases throughout the operating time. It reflects the GTE performance deterioration because of different failure mechanisms in the GTE parts and sub-system related to irreversible structural degradation. For PIs related to EGT, the majority of the values of the difference between $\mathrm{PI}_{\mathrm{j}}$ and PI1 are also negative, 
and indicates good long-term trends. From the plotted accumulated difference shown in Figure 8, it can be seen that throughout the operating period, even though overall decrease is observable for PIs related to Power, the change rates of them are not constant. More work can be performed to study the long-term performance deterioration patterns and develop prediction models to estimate impending failures.

\section{Conclusion}

Performance analysis for a low power rating and partially loaded gas turbine engine, is carried out by a proposed modelfree data analytics approach using available data. Several performance indices (PI) are proposed considering the ratio of Power and EGT output to combination of parameters related to fuel consumption. The effects of ambient temperature on PIs and the efficacy of using these PIs for identifying the short-term performance and long-term performance deterioration arising from compressor fouling and structural degradation respectively. Conclusions that emerge from the analysis of the obtained result are as follows:

- PIs using single as well as combined engine parameters show overall consistent dependence on the ambient temperature. The PIs related to Power increases along with ambient temperature up to around $288 \mathrm{~K}$, indicating the engine gets relatively better performance and high efficiency in this temperature range. The PI decreases for $\mathrm{T}_{\mathrm{amb}}$ higher than $288 \mathrm{~K}$. This is due to the effect of parasitic losses in the pre-cooler used for power augmentation observed during summer months.

- Correlation analysis based input selection supports the choices of PIs proposed in this paper. Also, based on this correlation analysis, one can conclude the PIs based on Power are more effective than those based on EGT for performance monitoring of gas turbine engines.

- A new analysis approach for short-term performance deterioration patterns is adopted by directly comparing the after-wash and before-wash values of PIs for every wash. The selected PIs especially related to Power shows good estimation of short-term deterioration throughout the operating period.

- A new method for long-term performance deterioration patterns is presented by directly comparing the after wash values of the PI for each wash time, eliminating the effect of the short-term deterioration and the influence of the ambient temperature. The results confirm that both new and old PIs related to Power consistently captures the trend of the long-term deterioration. While for the PIs related to EGT, only new PIs are able to capture such deterioration. This suggests the need of adopting the combination of parameters to define the new PIs.

- Comparing to other prognostic methods, this novel approach provides a simpler, cheaper way to analyze system performance, especially in real-time. The result demonstrates the feasibility of deployment of model-free data analytics using a systematic data manipulation methodology and confirms the applicability of the proposed performance indices for performance monitoring. The work can be continued with introduction of additional PIs for more comprehensive analysis and prediction for short-term and long-term performance deterioration, towards a holistic Prognostics and Health Management (PHM) system for gas turbine engine.

\section{REFERENCES}

Carter, T.J., (2005). Common Failures in Gas Turbine Blades. Engineering Failure Analysis, 12, 237-247.

Coble, J.B. and Hines, J.W., (2013). Identifying Suitable Degradation Parameters for Individual-Based. Prognostics. In S. Kadry (Ed.), Diagnostics and Prognostics of Engineering Systems: Methods and Techniques, 135-150.

Diakunchak, I.S., (1992). Performance Deterioration in Industrial Gas Turbines. Journal of Engineering for Gas Turbines and Power, 114(2), 161-168.

Feldman, A., De Castro, H.V., van Gemund, A., and Provan, G., (2013). Model-based Diagnostic Decision-support System for Satellites. Aerospace Conference, 2013 IEEE, 1-14.

Ghonemy A.M.K.E (2016).Gas Turbines Waste Heat/Power Recovery in Tropical Climate Zones: Analysis to Inform Decision Making. IOSR Journal of Mechanical and Civil Engineering, 13(3), 36-44.

Guyon, I., and Elisseeff, A., (2003). An Introduction to variable and feature selection. Journal of Machine Learning Research, 3, 1157-1182.

Hanachi, H., Alavi, E., Liu, J., Banerjee, A., Koul, A., and Liang, M., 2012. Bladed Disk Crack Detection through Advanced Analysis of Blade Time of Arrival Signal. Prognostics and Health Management (PHM), 2012 IEEE Conference, Denver, CO, USA, 18 Jun - 21 Jun 2012.

Hanachi, H., Liu, J., Banerjee, A., Chen, Y., Koul, A., (2014). A Physics-Based Modeling Approach for Performance Monitoring in Gas Turbine Engines. ASME Turbo Expo 2014: Turbine Technical Conference and Exposition: V006T06A021-V006T06A021.

Hanachi, H., Jie Liu, Avisekh Banerjee, Ying Chen, (2015). A Framework with Nonlinear System Model and Nonparametric Noise for Gas Turbine Degradation State Estimation, Measurement Science and Technology, 26(6), 065604.

Hyndman, R. J., \& Koehler, A. B. (2006). Another look at measures of forecast accuracy. International Journal of Forecasting, 22,679-688.

Kacprzynski G.J., Gumina, M., Roemer, M.J., Caguiat, D.E., Galie, T.R., McGroarty, J.J., (2001). A Prognostic 
Modeling Approach for Predicting Recurring Maintenance for Shipboard Propulsion Systems. ASME Turbo Expo 2001: Power for Land, Sea, and Air Volume 1: Aircraft Engine; Marine; Turbomachinery; Microturbines and Small Turbomachinery. New Orleans, Louisiana, USA, June 4-7, 2001.

Kurz, R., and Brun, K., (2009). Degradation of Gas Turbine Performance in Natural Gas Service. Journal of Natural Gas Science and Engineering, 1, 95-102.

Li, G. Y., (2002). Performance Analysis Based Gas Turbine Fault Diagnostics: A review. Proceedings of the Institution of Mechanical Engineers, Part A: Journal of Power and Energy, 216 (5), 363-377.

Li, C.J., and Lee, H., (2005). Gear Fatigue Crack Prognosis using Embedded Model, Gear Dynamic Model and Fracture Mechanics. Mechanical Systems and Signal Processing, 19, 836-846.

Li, Y.G., and Nilkitsaranont, P., (2009). Gas Turbine Performance Prognostic for Condition-Based Maintenance. Applied Energy, 86, 2152-2161.

Liu, Y., Banerjee, A., Kumar, A., Srivastava, A., and Goel, N., 2017. Effect of Ambient Temperature on Performance of Gas Turbine Engine. Annual Conference of the Prognostics and Health Management Society 2017, St. Petersburg, FL, USA, Oct 2-5, 2017.

Luo, J., Namburu, M., Pattipati, K.R., Qiao, L., and Chigusa, S., (2010). Integrated Model-based and Data-driven diagnosis of Automotive Antilock Braking Systems. Systems, Man and Cybernetics, Part A: Systems and Humans, IEEE Transactions On, 40, 321-336.

Meher-Homji, C.B., Chaker, M., and Bromley, A.F., (2009). The Fouling of Axial Flow Compressors: Causes, Effects, Susceptibility, and Sensitivity. ASME Turbo Expo 2009: Power for Land, Sea, and Air: 571-590.

Mohanty B., Paloso J., (1995). Enhancing gas turbine performance by intake air cooling using an absorption chiller, Heat Recovery System, CHPJ; 15(1), 41-50.

Namburu, S.M., Wilcutts, M., Chigusa, S., Qiao, L., Choi, K., and Pattipati, K., (2006). Systematic Data-driven Approach to Real-time Fault Detection and Diagnosis in automotive engines. Autotestcon, 2006 IEEE, 59-65.

Rai,A., and Kohli, A., (2012). Simulation and Analysis of Nonlinear System Identification using the LMS Volterra Filter. Advanced Materials Research, 3528-3537.

Sun, J., Zuo, H., Wang, W., and Pecht, M.G. (2012). Application of a State Space Modeling Technique to System Prognostics Based on a Health Index for Condition-Based Maintenance," Mechanical Systems and Signal Processing, 28(4), 585-596.

Yu, L., Cleary, D., Osborn, M., and Rajiv, V., (2007). Information Fusion Strategy for Aircraft Engine Health Management. ASME Turbo Expo 2007: Power for Land, Sea, and Air, 531-538.

\section{BIOGRAPHIES}

Dr. Yuan Liu is a senior research scientist with more than 15 years of research and consulting experience in the field of data analysis, prognostics and health management of gas turbine engine components, thermal dynamic and CFD simulation on combustion process inside combustor of gas turbine, multiphase flow.

Dr. Avisekh Banerjee is the Director of Engineering at Life Prediction Technologies Inc. His broad areas of interest and expertise lies in performing physics-based prognostics, ENSIP, data trending for failure prediction and analytics, design and development of PHM framework for varied applications. He liaises with the Clients and partners requiring engineering and consulting services, manages technical teams within LPTi and oversees R\&D collaborations. Dr. Banerjee is a registered professional engineer in Ontario, Canada.

Dr. Thambirajah Ravichandran is a Research Associate at the Department of Systems Design Engineering, University of Waterloo. Dr. Ravichandran obtained his PhD in Electrical \& Computer Engineering from the University of Waterloo. His broad areas of interest and expertise are in applying machine learning and computational intelligence techniques for the design and development of control systems and pattern recognition systems. Dr. Ravichandran is a registered professional engineer in Ontario, Canada.

Dr. Amar Kumar has more than 25 years of research and consulting experience in the fields of structural materials characterization and development, fracture mechanics, failure analysis and applications. Dr. Kumar is currently working as senior research scientist in the R\&D project of diagnostics, prognostics and health management of aeroengine components. He specializes in both data driven approaches and physics-based modeling and simulations.

Dr. Glenn Heppler is a graduate from the Institute for Aerospace Studies at the University of Toronto. His Ph.D. was in the area of finite element analysis of the behaviour of nonlinear dynamic structures. Professor Heppler has been at the Department of Systems Design Engineering, University of Waterloo since 1986 and has served two terms as department Chair. He is a member of the American Society for Mechanical Engineering. He regularly reviews papers for several international journals. Currently Dr. Heppler's research interests center on the dynamics and control of flexible structures, and gyroscopic systems as applied to MEMS devices. 